Pacific

Journal of

Mathematics

GAUSS MAP HARMONICITY AND MEAN CURVATURE OF A HYPERSURFACE IN A HOMOGENEOUS MANIFOLD

FiDELIS BITTENCOURT AND JAIME RIPOLL 


\title{
GAUSS MAP HARMONICITY AND MEAN CURVATURE OF A HYPERSURFACE IN A HOMOGENEOUS MANIFOLD
}

\author{
FIDELIS BITTENCOURT AND JAIME RIPOLL
}

\begin{abstract}
We define a Gauss map of an orientable hypersurface in a homogeneous manifold with an invariant Riemannian metric. Our main objective is to extend to this setting some results on the Gauss map of a constant mean curvature hypersurface of an Euclidean space, namely the Ruh-Vilm theorem relating the harmonicity of the Gauss map and the constancy of the mean curvature, and the Hoffman-Osserman-Schoen theorem characterizing the plane and the circular cylinder as the only complete constant mean curvature surfaces whose Gauss image is contained in a closed hemisphere of the sphere.
\end{abstract}

\section{Introduction}

In this article we define a Gauss map of an orientable hypersurface in a homogeneous manifold with an invariant Riemannian metric. Our main objective is to extend to this setting some results on the Gauss map of a constant mean curvature hypersurface of an Euclidean space. We focus our investigation on the extension of two well known theorems:

Theorem (Ruh-Vilm). A hypersurface in $\mathbb{R}^{n}$ has constant mean curvature if and only if the Gauss map of the hypersurface is harmonic.

Theorem (Hoffman-Osserman-Schoen). If the Gauss map of a complete constant mean curvature surface in $\mathbb{R}^{3}$ is contained in a closed hemisphere of the sphere, the surface is a plane or a circular cylinder.

Extensions of these results were already given in [Espírito-Santo et al. 2003] in the case that the ambient space is a Lie group with a bi-invariant metric; the main theorems in the present work extend those of that reference to the case of homogeneous spaces. We also obtain certain other results.

Section 2 introduces notations and basic facts about homogeneous manifolds which will be used throughout the paper. We introduce some of these notations

MSC2000: 53A10.

Keywords: Gauss map, harmonic map, mean curvature. 
now. Let $\mathbb{G}$ be a Lie group with a fixed bi-invariant Riemannian metric, $\mathbb{H}$ a compact Lie subgroup of $\mathbb{G}$. Set $k=\operatorname{dim} \mathbb{H}$ and $n+k+1=\operatorname{dim} \mathbb{G}$. In the quotient $\mathbb{G} / \mathbb{H}$ of left residue classes we consider a homogeneous Riemannian metric in such a way that the projection $\pi: \mathbb{G} \rightarrow \mathbb{G} / \mathbb{H}$ becomes a Riemannian submersion. Denote by $\mathscr{G}$ the Lie algebra of $\mathbb{G}$, by $\mathscr{H}$ the Lie algebra of $\mathbb{H}$, and let $\mathbb{S}^{n+k}$ be the unit sphere centered at the origin of $\mathscr{G}$. Let $M$ be an orientable hypersurface in $\mathbb{G} / \mathbb{H}$ oriented by a unit normal vector field $\eta$.

In Section 3 we define, prove some general properties of, and give examples of the Gauss map $N: M \rightarrow \mathbb{S}^{n+k}$ of $M$; this map is defined by taking the horizontal lifting $\tilde{\eta}$ of $\eta$ to $\mathbb{G}$ followed by the right translation of $\tilde{\eta}$ to the identity of $\mathbb{G}$, that is, the right translation of $\tilde{\eta}$ to $\mathscr{G}$. In the Euclidean space, taking $\mathbb{G}=\mathbb{R}^{n}$ and $\mathbb{U}=\{e\}$, $N$ coincides with the usual Gauss map of $M$.

In Section 4 we study the Gauss map of constant mean curvature hypersurfaces of $\mathbb{G} / \mathbb{H}$. Our main result asserts that $M$ has constant mean curvature if and only if $N$ is harmonic (Corollary 4.4). This fact has many implications and, to state some of them, we first introduce a definition.

A codimension one vector subspace of $\varphi$ divides $\varphi$ in two connected components; the closure of any of these components is called a half-space of $\varphi$ and its intersection with $\mathbb{S}^{n+k}$ is a half-sphere, denoted by $\mathbb{S}_{+}^{n+k}$. Given $l \in \mathbb{N}$, a $\left(1 / 2^{l}\right)$ sphere $\mathbb{S}^{n+k, l}$ of $\mathbb{S}^{n+k}$ is the intersection of $l$ linearly independent half spheres $\left(\mathbb{S}_{1}^{n+k}\right)_{+}, \ldots,\left(\mathbb{S}_{l}^{n+k}\right)_{+}$of $\mathbb{S}^{n+k}$ (by "linearly independent" we mean that the normal vectors to the bounding half-spaces are linearly independent). We prove:

Let $M$ be a compact constant mean curvature hypersurface of $\mathbb{G} / \mathbb{E}$ and $N: M \rightarrow$ $\mathbb{S}^{n+k}$ the Gauss map of $M$. Assume that either $M$ is not totally geodesic or the Ricci curvature of $\mathbb{G}$ is positive. Given $l \in \mathbb{N}$, let

$$
\mathbb{S}^{n+k, l}=\bigcap_{i=1}^{l}\left(\mathbb{S}_{i}^{n+k}\right)_{+}
$$

be a $\left(1 / 2^{l}\right)$-sphere of $\mathbb{S}^{n+k}$. Then there is equivalence between:

(1) $N(M) \subset \mathbb{S}^{n+k, l}$.

(2) $N(M) \subset \bigcap_{i=1}^{l} \partial\left(\mathbb{S}_{i}^{n+k}\right)_{+}$.

(3) $\mathscr{K}:=\left(\bigcap_{i=1}^{l} \partial\left(\mathbb{S}_{i}^{n+k}\right)_{+}\right)^{\perp}$ is a Lie subalgebra of $\mathscr{G}$ and $M$ is invariant under the Lie subgroup $\mathbb{K}$ of $\mathbb{G}$ whose Lie algebra is $\mathscr{K}$.

It follows under the same general hypotheses that if $l \geq n$, then $M$ is an extrinsically homogeneous manifold (that is, there is a Lie subgroup of isometries of $\mathbb{G} / \mathbb{W}$ acting transitively on $M$ ).

If $M$ is assumed only to be complete, a similar result holds when $\operatorname{dim}(\mathbb{G} / \mathbb{W})=3$. In this case, more explicit applications are given in Corollaries 4.13 and 4.14. In 
particular, we obtain the following result related to a conjecture of B. Lawson (which asserts that an embedded minimal torus in $\mathbb{S}^{3}$ has to be a Clifford torus, that is, the Riemannian product of two circles): A complete minimal surface $M$ in $\mathbb{S}^{3}=\mathrm{SO}(4) / \mathrm{SO}(3)$ is a Clifford torus if and only if the Gauss image $N(M)$ of $M$ lies in a certain hemisphere of the unit sphere through the origin in $\mathfrak{s o}(4)$ (Corollary 4.14).

The harmonicity of the Gauss map of a constant mean curvature surface can also be used to establish a stability criterion: If $D$ is a domain of a constant mean curvature surface $M$ of $\mathbb{G} / \mathbb{H}(\operatorname{dim}(\mathbb{G} / \mathbb{M})=3)$ such that $N(\bar{D})$ is contained in a half space of $G$, then $D$ is stable (Theorem 4.10).

\section{Preliminaries}

Let $\mathbb{G}$ be an $(n+k+1)$-dimensional connected Lie group, for $n \geq 2, k \geq 0$, with a bi-invariant Riemannian metric $\langle$,$\rangle . Denote by \widetilde{\nabla}$ the Riemannian connection on $\mathbb{G}$ determined by $\langle$,$\rangle . Let \mathscr{G}$ be the Lie algebra of $\mathbb{G}$. Given $x \in \mathbb{G}$, let $L_{x}$ and $R_{x}$ denote the left and right translations on $\mathbb{G}$, that is, $L_{x}(y)=x y$ and $R_{x}(y)=y x$. As usual, $\exp : \mathscr{G} \rightarrow \mathbb{G}$ denotes the Lie exponential map. Given $g \in \mathbb{G}$ and $X \in \mathscr{G}$, we have the maps $\operatorname{Ad}_{g}, \operatorname{ad}_{X}: \mathscr{G} \rightarrow \mathscr{G}$ defined by

$$
\operatorname{Ad}_{g}=d\left(R_{g}^{-1} \circ L_{g}\right)_{e} \quad \text { and } \quad \operatorname{ad}_{X}(Y)=[X, Y] .
$$

We note that

$$
\operatorname{ad}_{X}(Y)=\left.\frac{d}{d t} \operatorname{Ad}_{\exp t X}(Y)\right|_{t=0},
$$

and that $\mathrm{Ad}_{g}$ is a Lie group isomorphism and an isometry.

We recall that if $X, Y, Z$ are both left (or both right) invariant vector fields of $\mathbb{G}$, then $\widetilde{\nabla}$ and the curvature tensor $R$ on $\mathbb{G}$ are given by

$$
\widetilde{\nabla}_{X} Y=\frac{1}{2}[X, Y] \quad \text { and } \quad R(X, Y) Z=\frac{1}{4}[[X, Y], Z] .
$$

We have $\langle[X, Y], Z\rangle=\langle[Z, Y], X\rangle$. The Ricci tensor of $\mathbb{G}$ is given by

$$
\operatorname{Ric}(u, v)=\operatorname{trace}(X \mapsto R(u, X) v)=\sum_{i=1}^{n+k+1}\left\langle R\left(u, E_{j}\right) v, E_{j}\right\rangle,
$$

where $\left\{E_{j}\right\}$ is an orthonormal basis of tangent vectors of $\mathbb{G}$. The Ricci curvature of $\mathbb{G}$ on the $u$-direction is $\operatorname{Ric}(u)=\operatorname{Ric}(u, u)$. Throughout this article, Ric will always mean the Ricci curvature of $\mathbb{G}$.

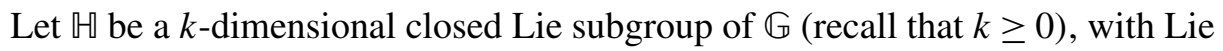
algebra $\mathscr{H}$, and let $\mathbb{G} / \mathbb{H}$ be the smooth $(n+1)$-dimensional manifold of left residue classes of $\mathbb{H}$ :

$$
\mathbb{G} / \mathbb{H}=\{x \mathbb{M}: x \in \mathbb{G}\} .
$$


We consider on $\mathbb{G} / \mathbb{W}$ a Riemannian metric, also denoted by $\langle$,$\rangle , induced by the$ projection

$$
\pi: \mathbb{G} \rightarrow \mathbb{G} / \mathbb{H}, \quad \pi(x)=x \mathbb{H},
$$

as follows. Given $z \in \mathbb{G} / \mathbb{H}$, take $x \in \pi^{-1}(z)$ and define an inner product $\langle$,$\rangle in$ $T_{z}(\mathbb{G} / \mathbb{H})$ in such a way that

$$
l_{x}:=\left.d \pi_{x}\right|_{T_{x}\left(\pi^{-1}(z)\right)^{\perp}}: T_{x}\left(\pi^{-1}(z)\right)^{\perp} \rightarrow T_{z}(\mathbb{G} / \mathbb{Q})
$$

is a linear isometry, where ${ }^{\perp}$ denotes the orthogonal complement in $T_{x}(\mathbb{G})$. It is easy to see that $\langle$,$\rangle is well defined in T_{z}(\mathbb{G} / \mathbb{W}$ ) (that is, it does not depend on the choice of $\left.x \in \pi^{-1}(z)\right)$ and defines a Riemannian homogeneous (bi-invariant) metric on $\mathbb{G} / \mathbb{W}$. Denote by $\nabla$ the Riemannian connection associated to $\langle$,$\rangle .$

Denote by $\mathscr{L}(\mathbb{G} / \mathbb{M})$ and by $\mathscr{X}(\mathbb{G})$ the spaces of smooth vector fields on $\mathbb{G} / \mathbb{M}$ and $\mathbb{G}$. A vector field $\widetilde{X}$ in $\mathbb{G}$ is called horizontal if $\widetilde{X}(x) \in T_{x}(x \mathbb{H})^{\perp}$ for $x \in \mathbb{G}$, and is

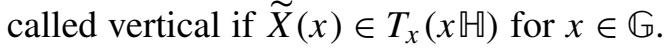

Given $\widetilde{X} \in \mathscr{L}(\mathbb{G})$, let $\widetilde{X}^{v}$ be the vertical vector field on $\mathbb{G}$ given as the orthogonal projection of $\tilde{X}(x)$ on $T_{x}(x \mathbb{H})$, and let $\widetilde{X}^{h}$ be the horizontal vector field on $\mathbb{G}$ given as the orthogonal projection of $\widetilde{X}(x)$ on $T_{x}(x \mathbb{U})^{\perp}, x \in \mathbb{G}$.

It follows forthwith from the definition of $\langle$,$\rangle that the projection \pi: \mathbb{G} \rightarrow \mathbb{G} / \mathbb{M}$ is a Riemannian submersion. It is not difficult to see that given $X, Y \in \mathscr{X}(\mathbb{G} / \mathbb{U})$, we have

$$
\widetilde{\nabla}_{\widetilde{X}} \tilde{Y}(x)=l_{x}^{-1}\left(\nabla_{X} Y(\pi(x))\right)+\frac{1}{2}[\tilde{X}, \widetilde{Y}]^{v}(\pi(x)),
$$

where $\tilde{X}, \tilde{Y} \in \mathscr{X}(\mathbb{G})$ are defined by $\tilde{X}(x)=l_{x}^{-1}(X(\pi(x)))$ and $\tilde{Y}(x)=l_{x}^{-1}(Y(\pi(x)))$ for $x \in \mathbb{G}$, and [ , ] is the Lie bracket in $\mathbb{G}$.

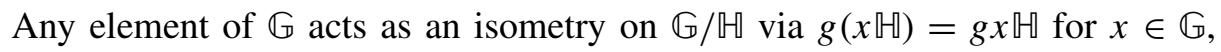
$g \in \mathbb{G}$, or

$$
g(\pi(x))=\pi\left(R_{x}(g)\right) .
$$

We may then consider $\mathbb{G}$ as a Lie subgroup of the isometry group Iso $(\mathbb{G} / \mathbb{H})$ of $\mathbb{G} / \mathbb{H}$. It follows that any vector $w \in \mathscr{G}$ acts on $\mathbb{G} / \mathbb{H}$ as a Killing vector field, which we denote by $\zeta(w)$ :

$$
\zeta(w)(z)=\left.\frac{d}{d t}(\exp t w)(z)\right|_{t=0} \text { for } z \in \mathbb{G} / \mathbb{H} .
$$

Given $z \in \mathbb{G} / \mathbb{H}$ and $x \in \pi^{-1}(z)$, we have, using (2-1)

$$
\begin{aligned}
\zeta(w)(z) & =\left.\frac{d}{d t}(\exp t w)(z)\right|_{t=0}=\left.\frac{d}{d t}(\exp t w)(\pi(x))\right|_{t=0} \\
& =\left.\frac{d}{d t} \pi\left(R_{x}(\exp t w)\right)\right|_{t=0}=d \pi_{x}\left(d\left(R_{x}\right)_{e}(w)\right),
\end{aligned}
$$


that is

$$
\zeta(w)(z)=d \pi_{x}\left(d\left(R_{x}\right)_{e}(w)\right) .
$$

Note that $\zeta: \mathscr{G} \rightarrow \mathscr{X}(\mathbb{G} / \mathbb{W})$ is a Lie algebra monomorphism.

From now on, unless otherwise stated, we keep the assumptions and notations of this section concerning the Lie groups $\mathbb{G}$ and $\mathbb{H}$.

\section{The Gauss map of a hypersurface in $\mathbb{G} / \mathbb{W}$}

We begin by introducing a "translation" $\Gamma$ in $\mathbb{G} / \mathbb{H}$. Given $z \in \mathbb{G} / \mathbb{H}$, define a map

$$
\Gamma_{z}: T_{z}(\mathbb{G} / \mathbb{H}) \rightarrow \mathscr{G}
$$

by choosing $x \in \pi^{-1}(z)$ and setting

$$
\Gamma_{z}(u):=d\left(R_{x}\right)_{x}^{-1}\left(l_{x}^{-1}(u)\right) .
$$

Proposition 3.1. The map $\Gamma_{z}$ is well-defined, that is, (3-1) does not depend on $x \in \pi^{-1}(z)$. Moreover, $\Gamma_{z}: T_{z}(\mathbb{G} / \mathbb{Q}) \rightarrow \mathscr{G}$ is linear and preserves the metric.

Proof. Consider $x, y \in \pi^{-1}(z)$ and $u \in T_{z}(\mathbb{G} / \mathbb{M})$. Let $h \in \mathbb{U}$ be such that $x=y h$. We obviously have $d\left(R_{h}\right)_{y}\left(T_{y} \pi^{-1}(z)\right)=T_{x} \pi^{-1}(z)$ and, since $R_{h}$ is an isometry, $d\left(R_{h}\right)_{y}\left(T_{y} \pi^{-1}(z)^{\perp}\right)=T_{x} \pi^{-1}(z)^{\perp}$. It follows that $d g\left(R_{h}\right)_{y}\left(l_{x}^{-1}(u)\right) \in\left(T_{x} \pi^{-1}(z)\right)^{\perp}$. Observing that

$$
l_{x}\left(d\left(R_{h}\right)_{y}\left(l_{y}^{-1}(u)\right)\right)=d\left(\pi \circ R_{h}\right)_{y}\left(l_{x}^{-1}(u)\right)=d \pi_{y}\left(l_{y}^{-1}(u)\right)=u=d \pi_{x}\left(l_{x}^{-1}(u)\right),
$$

so that $d g\left(R_{h}\right)_{y}\left(l_{y}^{-1}(u)\right)=l_{x}^{-1}(u)$, we have

$$
\begin{aligned}
d g\left(R_{x}\right)_{x}^{-1}\left(l_{x}^{-1}(u)\right) & =d\left(R_{x^{-1}}\right)_{x}\left(d g\left(R_{h}\right)_{y}\left(l_{y}^{-1}(u)\right)\right) \\
& =d g\left(R_{x^{-1}} \circ R_{h}\right)_{y}\left(l_{y}^{-1}(u)\right)=d g\left(R_{y^{-1}}\right)_{y}\left(l_{y}^{-1}(u)\right),
\end{aligned}
$$

proving that $\Gamma_{z}$ is well defined. Moreover, $\Gamma_{z}$ is obviously linear and, since $l_{x}$ and $R_{x}^{-1}$ are isometries, $\Gamma_{z}$ preserves the metric.

The generalized Gauss map. Let $M$ be an immersed, orientable hypersurface of $\mathbb{G} / \mathbb{H}$. Let $\eta$ be a unit normal vector field to $M$ in $\mathbb{G} / \mathbb{H}$. We define the Gauss map $N$ of $M$ as the map

$$
N: M^{n} \rightarrow \mathbb{S}^{n+k} \subset \mathcal{G} \text { given by } N(z)=\Gamma_{z}(\eta(z)) .
$$

A family of examples. Let $\mathbb{K}$ be a compact Lie subgroup of $\mathbb{G}$ and $\mathscr{K}$ its Lie algebra.

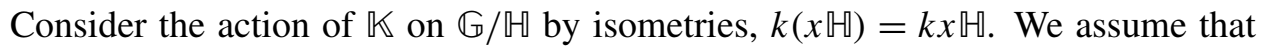
this is a cohomogeneity-one action, that is, the orbits of $\mathbb{K}$ of highest dimension have codimension 1 in $\mathbb{G} / \mathbb{H}$. 
Let $g \in \mathbb{G}$ be such that

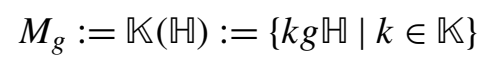

is an orbit of $\mathbb{K}$ of codimension 1 . Then $M_{g}$ is a compact orientable embedded hypersurface of $\mathbb{G} / \mathbb{H}$.

Lemma 3.2. The vector subspace $\mathrm{Ad}_{k g}(\mathscr{H})+\mathscr{K}$ has codimension 1 in $\mathscr{G}$, for any $k \in \mathbb{K}$.

Proof. Set $\widetilde{M}_{g}:=\pi^{-1}\left(M_{g}\right)$. Then $\widetilde{M}_{g}$ is an embedded hypersurface of $\mathbb{G}$ (possibly

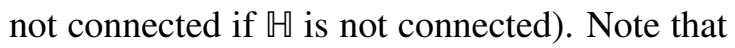

$$
\tilde{M}_{g}=\{k g h \mid k \in \mathbb{K}, h \in \mathbb{U}\}
$$

and it follows that $T_{x}\left(\widetilde{M}_{g}\right)=d\left(L_{x}\right)_{e}(\mathscr{H})+d\left(R_{x}\right)_{e}(\mathscr{K})$ for any $x \in \tilde{M}_{g}$, so that

$$
\operatorname{Ad}_{x}(\mathscr{H})+\mathscr{K}=d\left(R_{x}^{-1}\right)_{x}\left(T_{x}\left(\tilde{M}_{g}\right)\right)
$$

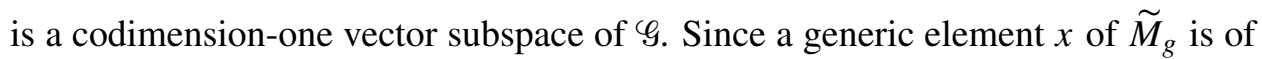
the form $x=k g h$, we have $\operatorname{Ad}_{x}(\mathscr{H})=\left(\operatorname{Ad}_{k} \circ \operatorname{Ad}_{g} \circ \operatorname{Ad}_{h}\right)(\mathscr{H})=\operatorname{Ad}_{k g}(\mathscr{H})$, so that

$$
\operatorname{Ad}_{k g}(\mathscr{H})+\mathscr{K}=d\left(R_{x}^{-1}\right)_{x}\left(T_{x}\left(\tilde{M}_{g}\right)\right)
$$

is a codimension-1 vector subspace of $\mathscr{G}$.

Proposition 3.3. Set $N_{0}=N(g \llbracket)$, where $N: M_{g} \rightarrow \mathbb{S}^{n+k}$ is the Gauss map of $M_{g}$. Then

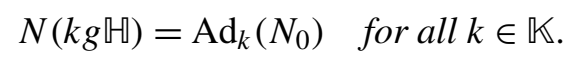

Proof. Let $\eta$ be a unit normal vector field to $M_{g}$ defining $N$. Given $z=k g \llbracket \in M_{g}$, choose $x \in \widetilde{M}_{g}=\pi^{-1}(M), x=k g h$. Then note that $l_{x}^{-1}(\eta(z))$ is orthogonal to $T_{x}\left(\tilde{M}_{g}\right)$ so that, by definition of $N$,

$$
N(k g \llbracket)=N(z)=d\left(R_{x}^{-1}\right)_{x}\left(l_{x}^{-1}(\eta(z))\right)
$$

is orthogonal to

$$
d\left(R_{x}^{-1}\right)_{x}\left(T_{x}\left(\tilde{M}_{g}\right)\right)=\operatorname{Ad}_{k g}(\mathscr{H})+\mathscr{K} .
$$

In particular, $N_{0}=N(g \llbracket)$ is orthogonal to $\operatorname{Ad}_{g}(\mathscr{H})+\mathscr{K}$. Then $\operatorname{Ad}_{k}\left(N_{0}\right)$ is orthogonal to

$$
\operatorname{Ad}_{k}\left(\operatorname{Ad}_{g}(\mathscr{H})+\mathscr{K}\right)=\operatorname{Ad}_{k g}(\mathscr{H})+\operatorname{Ad}_{k}(\mathscr{K})=\operatorname{Ad}_{k g}(\mathscr{H})+\mathscr{K}
$$

and $N(k g \llbracket)$ and $\operatorname{Ad}_{k}\left(N_{0}\right)$ are both orthogonal to $\operatorname{Ad}_{k g}(\mathscr{H})+\mathscr{K}$. Since

$$
\operatorname{dim}\left(\operatorname{Ad}_{k g}(\mathscr{H})+\mathscr{K}\right)=\operatorname{dim} \mathscr{G}-1,
$$

we have

$$
N(z)=N(k g \llbracket)= \pm \operatorname{Ad}_{k}\left(N_{0}\right) .
$$

By continuity, $N(k g \llbracket)=\operatorname{Ad}_{k}\left(N_{0}\right)$, since $N(g \llbracket)=N_{0}=\operatorname{Ad}_{e}\left(N_{0}\right)$ at $k=e$. 
Remark. As we see from the proof, the vector $N_{0}$ quoted on the statement of Proposition 3.3 can be easily determined, since it is orthogonal to the codimension1 subspace $\operatorname{Ad}_{g}(\mathscr{H})+\mathscr{K}$.

The next result gives a characterization of the homogeneous hypersurfaces of $\mathbb{G} / \mathbb{W}$ in terms of the Gauss map. This proposition is important for the characterization of the constant mean curvature hypersurfaces given in Section 4.

Proposition 3.4. Let $M$ be an orientable hypersurface of $\mathbb{G} / \mathbb{H}$ and let $N: M \rightarrow$ $\mathbb{S}^{n+k}$ be the Gauss map of M. Then

$$
\mathscr{K}:=N(M)^{\perp}=\{w \in \mathscr{G} \mid\langle w, v\rangle=0 \text { for all } v \in N(M)\}
$$

is a Lie subalgebra of $G$ and $M$ is invariant under the Lie subgroup of $\mathbb{G}$ whose Lie algebra is $\mathscr{K}$. In particular, if $\operatorname{dim} \mathscr{K}=n=\operatorname{dim}(\mathbb{G} / \mathbb{H})-1$, then $M$ is an extrinsically homogeneous submanifold of $\mathbb{G} / \mathbb{H}$.

Conversely, if $M$ is invariant under a Lie subgroup $\mathbb{K}$ of $\mathbb{G}$, then $\mathscr{K} \subset N(M)^{\perp}$, where $\mathscr{K}$ is the Lie algebra of $\mathbb{K}$.

Lemma 3.5. If $w \in N(z)^{\perp}$ then $\zeta(w)(z) \in T_{z} M$.

Proof. If $w \in N(z)^{\perp}$ then

$$
\begin{aligned}
0 & =\langle w, N(z)\rangle=\left\langle d\left(R_{x}\right)_{e} w, l_{x}^{-1}(\eta)\right\rangle \\
& =\left\langle d \pi_{x}\left(d\left(R_{x}\right)_{e}\right) w, d \pi_{x}\left(l_{x}^{-1}(\eta)\right)\right\rangle=\langle\zeta(w)(z), \eta(x)\rangle
\end{aligned}
$$

so that $\zeta(w)(z) \in T_{z} M$, proving the lemma.

Proof of Proposition 3.4. Let $v, w \in N(M)^{\perp}$ be given. It follows by Lemma 3.5 that $\zeta(v)$ and $\zeta(w)$ are vector fields on $M$ so that $[\zeta(v), \zeta(w)]$ is a vector field on $M$. Since $\zeta$ is a Lie monomorphism, $[\zeta(v), \zeta(w)]=\zeta([v, w])$. Then, given $z \in M$,

$$
\begin{aligned}
0 & =\langle\zeta([v, w]), \eta\rangle=\left\langle\Gamma_{z}(\zeta([v, w])), \Gamma_{z}(\eta)\right\rangle \\
& =\left\langle d\left(R_{x}^{-1}\right)_{x}\left(l_{x}^{-1}\left(d \pi_{x}\left(d\left(R_{x}\right)_{e}([v, w])\right)\right)\right), N(z)\right\rangle \\
& =\left\langle d\left(R_{x}^{-1}\right)_{x}\left(d\left(R_{x}\right)_{e}([v, w])^{h}\right), d\left(R_{x}^{-1}\right)_{x}\left(l_{x}^{-1}(\eta)\right)\right\rangle \\
& =\left\langle\left(d\left(R_{x}\right)_{e}([v, w])\right)^{h}, l_{x}^{-1}(\eta)\right\rangle \\
& =\left\langle d\left(R_{x}\right)_{e}([v, w]), l_{x}^{-1}(\eta)\right\rangle=\left\langle[v, w], d\left(R_{x}^{-1}\right)_{x} l_{x}^{-1}(\eta)\right\rangle=\langle[v, w], N(z)\rangle .
\end{aligned}
$$

Since $z$ is arbitrary, we get $[v, w] \in N(M)^{\perp}$, proving that $N(M)^{\perp}$ is a Lie subalgebra of $\mathscr{G}$. Conversely, if $M$ is $\mathbb{K}$-invariant then, given $w \in \mathcal{K}$, it follows that $\zeta(w)$ is a vector field on $M$, so that $\langle\zeta(w), \eta\rangle=0$ on $M$. We then have, as above,

$$
0=\langle\zeta(w), \eta\rangle=\left\langle\Gamma_{z}(\zeta(w)), \Gamma_{z}(\eta)\right\rangle=\langle w, N(z)\rangle
$$

proving that $\mathscr{K} \subset N(M)^{\perp}$. 


\section{The harmonicity of the Gauss map and the mean curvature}

It is well known that a hypersurface of $\mathbb{R}^{n+1}$ has constant mean curvature if and only if its Gauss map is harmonic. Our main purpose in this section is to extend this result to hypersurfaces of a homogeneous manifold. We recall that in $\mathbb{R}^{n+1}$, this equivalence between constant mean curvature hypersurfaces and the harmonicity of the Gauss map is an immediate corollary of the well known formula

$$
\Delta N=-\operatorname{grad} H-\|B\|^{2} N .
$$

This formula was extended to hypersurfaces in a Lie group in [Espírito-Santo et al. 2003] and, more generally, using Killing vector fields, to Killing parallelizable Riemannian manifolds; see [Fornari and Ripoll 2004]. Our objective now is to extend (4-1) to homogeneous manifolds using the Gauss map defined here.

Let $\mathbb{G}, \mathbb{H}$ and $\mathbb{G} / \mathbb{W}$ be as before and let $M$ be an immersed orientable hypersurface in $\mathbb{G} / \mathbb{H}$. Consider the Gauss map $N: M \rightarrow \mathbb{S}^{n+k} \subset \mathscr{G}$. Let

$$
\left\{e_{1}, e_{2}, \ldots, e_{n+k+1}\right\} \subset \varphi
$$

be an orthonormal basis of $\mathscr{G}$, and define functions $N_{i}: M \rightarrow \mathbb{R}$ by setting $N(z)=$ $\sum_{i=1}^{n+k+1} N_{i}(z) e_{i}$ for $z \in M$. The Laplacian of $N$ is defined by

$$
\Delta N:=\sum_{i=1}^{n+k+1} \Delta N_{i} e_{i},
$$

where $\Delta N_{i}$ is the usual Laplacian of $N_{i}$ with respect to the metric on $M$ induced by the immersion of $M$ in $\mathbb{G} / \mathbb{H}$. Then $N$ is harmonic if and only if

$$
\Delta_{\mathbb{S}^{n+k}} N=(\Delta N)^{\top}=0,
$$

where $^{\top}$ denotes the orthogonal projection of $\varphi$ on $T \mathbb{S}^{n+k}$ [Eells and Sampson 1964].

To get a proper extension of (4-1) to $\mathbb{G} / \mathbb{H}$, we need to introduce a new algebraicgeometric invariant of $M$ on $\mathbb{G} / \mathbb{H}$, a kind of Lie invariant second fundamental form (to appear later in Lemma 4.2). Given $z \in M$, define

$$
B_{z}^{i}(u)=\frac{1}{\sqrt{2}}\left(\operatorname{ad}_{N(z)}\left(\Gamma_{z}(u)\right)\right)^{v} \quad \text { for } u \in T_{z} M
$$

Lemma 4.1. Let $M$ be a hypersurface in $\mathbb{G} / \mathbb{H}$ and set $\widetilde{M}=\pi^{-1}(M)$. Let $f: M \rightarrow \mathbb{R}$ be a differentiable function and set $\tilde{f}=f \circ \pi: \widetilde{M} \rightarrow \mathbb{R}$. Then

$$
\Delta_{\tilde{M}} \tilde{f}=\left(\Delta_{M} f\right) \circ \pi .
$$


Proof. Choose $x_{0} \in \tilde{M}$ and set $z_{0}=\pi\left(x_{0}\right)$. Let $\left\{E_{i}\right\}, i=1, \ldots, n$, be an orthonormal frame on a neighborhood $V$ of $z_{0}$ in $M$. Set $W=\pi^{-1}(V)$ and define $\widetilde{E}_{i}$ on $W$, by

$$
\widetilde{E}_{i}(x)=l_{x}^{-1}\left(E_{i}(\pi(x)) .\right.
$$

Let $e_{i}, i=n+1, \ldots, n+k$, be an orthonormal basis of $\mathscr{H}$ and let $\widetilde{E}_{i}$ be the leftinvariant vector fields on $\mathbb{G}$ such that $\widetilde{E}_{i}(e)=e_{i}$. We have

$$
\widetilde{\nabla}_{\widetilde{E}_{i}} \widetilde{E}_{i}=\frac{1}{2}\left[\widetilde{E}_{i}, \widetilde{E}_{i}\right]=0
$$

for $i=n+1, \ldots, n+k$, and $\left\{\widetilde{E}_{i}\right\}_{i=1}^{n+k}$ is an orthonormal basis of $T_{x} \tilde{M}$ for $x$ in $W$.

We have

Since

$$
\Delta_{\widetilde{M}} \tilde{f}\left(x_{0}\right)=\sum_{i=1}^{n+k}\left\langle\widetilde{\nabla}_{\widetilde{E}_{i}} \operatorname{grad} \tilde{f}, \widetilde{E}_{i}\right\rangle
$$

$$
\widetilde{E}_{i}(\tilde{f})(x)=\widetilde{E}_{i}(f \circ \pi)(x)=d g(f \circ \pi)_{x}\left(\widetilde{E}_{i}(x)\right)=d f_{\pi(x)}\left(d \pi_{x}\left(\widetilde{E}_{i}(x)\right)\right)
$$

and, since $d \pi_{x}\left(\widetilde{E}_{i}(x)\right)=0$ for $i=n+1, \ldots, n+k$, we have $\widetilde{E}_{i}(\tilde{f})(x)=0$ for $i=n+1, \ldots, n+k$. Moreover, $\left.d \pi_{x}\left(\widetilde{E}_{i}(x)\right)\right)=E_{i}(\pi(x))$ for $i=1, \ldots, n$ so that $\widetilde{E}_{i}(\tilde{f})(x)=E_{i}(f)(\pi(x)), i=1, \ldots, n$, and we have

$$
\operatorname{grad} \tilde{f}(x)=\sum_{i=1}^{n+k} \widetilde{E}_{i}(\tilde{f})(x) \widetilde{E}_{i}(x)=\sum_{i=1}^{n} E_{i}(f)(\pi(x)) \widetilde{E}_{i}(x)
$$

so that $\operatorname{grad} \tilde{f}(x)=l_{x}^{-1}(\operatorname{grad} f(\pi(x)))$. Moreover, for $i=n+1, \ldots, n+k$,

$$
\left\langle\widetilde{\nabla}_{\widetilde{E}_{i}} \operatorname{grad} \tilde{f}, \widetilde{E}_{i}\right\rangle=\widetilde{E}_{i}\left\langle\operatorname{grad} \tilde{f}, \widetilde{E}_{i}\right\rangle-\left\langle\operatorname{grad} \tilde{f}, \widetilde{\nabla}_{\widetilde{E}_{i}} \widetilde{E}_{i}\right\rangle=0
$$

so that

$$
\begin{aligned}
\Delta_{\tilde{M}} \tilde{f}\left(x_{0}\right) & =\sum_{i=1}^{n}\left\langle\widetilde{\nabla}_{\widetilde{E}_{i}} \operatorname{grad} \tilde{f}, \widetilde{E}_{i}\right\rangle \\
& =\sum_{i=1}^{n}\left\langle l_{x}^{-1}\left(\nabla_{E_{i}} \operatorname{grad} f\right), \widetilde{E}_{i}\right\rangle+\frac{1}{2} \sum_{i=1}^{n}\left\langle\left[\widetilde{E}_{i}, \operatorname{grad} \tilde{f}\right]^{v}, \widetilde{E}_{i}\right\rangle \\
& =\sum_{i=1}^{n}\left\langle\nabla_{E_{i}} \operatorname{grad} f, E_{i}\right\rangle=\Delta_{M} f\left(\pi\left(x_{0}\right)\right),
\end{aligned}
$$

proving the lemma.

Lemma 4.2. Let $M$ be a hypersurface in $\mathbb{G} / \mathbb{H}$ and set $\tilde{M}:=\pi^{-1}(M)$. Denote by $\|B\|(\|\widetilde{B}\|)$ the norm of the second fundamental form $B(\widetilde{B})$ of $M(\widetilde{M})$ in $\mathbb{G} / \mathbb{U}(\mathbb{G})$, and by $H(\tilde{H})$ the mean curvature of $M(\tilde{M})$ with respect to a normal vector field $\eta\left(\tilde{\eta}=l_{x}^{-1} \eta\right)$. Suppose $\operatorname{dim} \mathbb{G}=n+k+1$ and $\operatorname{dim} \mathbb{H}=k$. 
(i) $\widetilde{H}(x)=\frac{n}{n+k} H(z)$ for all $z \in M$ and $x \in \pi^{-1}(z)$.

(ii) $\operatorname{grad} \widetilde{H}(x)=\frac{n}{n+k} l_{x}^{-1}(\operatorname{grad} H(z))$ for all $z \in M$ and $x \in \pi^{-1}(z)$.

(iii) $\|\widetilde{B}\|=\sqrt{\|B\|^{2}+\left\|B^{i}\right\|^{2}}$.

Proof. Choose $z \in M$ and $x \in \pi^{-1}(z)$. Let $E_{i}(z), i=1, \ldots, n$, be an orthonormal basis of $T_{z} M$ and define $\widetilde{E}_{i}(x)=l_{x}^{-1}\left(E_{i}(z)\right)$ for $i=1, \ldots, n$. Let $\widetilde{E}_{i}$, $i=n+2, \ldots, n+k+1$, be left-invariant vector fields on $\mathbb{G}$ such that $\widetilde{E}_{i}(x) \in T_{x} \widetilde{M}$ and $\left\{\widetilde{E}_{i}\right\}, i=1, \ldots, n+k+1$ is an orthonormal basis of $T_{x} \widetilde{M}$. Note that since $\widetilde{M}$ is 円-invariant, $\widetilde{E}_{i}$ is a vector field on $\widetilde{M}$ for $i=n+2, \ldots, n+k+1$. It is also easy to see that $\tilde{\eta}$ is a unit normal vector field to $\widetilde{M}$. It follows that, for $i, j=n+2, \ldots, n+k+1$,

$$
\left\langle\widetilde{\nabla}_{\widetilde{E}_{i}} \tilde{\eta}(x), \widetilde{E}_{j}(x)\right\rangle=-\left\langle\widetilde{\nabla}_{\widetilde{E}_{i}} \widetilde{E}_{j}(x), \tilde{\eta}(x)\right\rangle=-\frac{1}{2}\left\langle\tilde{\eta}(x),\left[\widetilde{E}_{i}, \widetilde{E}_{j}\right]\right\rangle=0
$$

since $\left[\widetilde{E}_{i}, \widetilde{E}_{j}\right]$ is a vector field on $\tilde{M}$.

Proof of (i). By the definition of the mean curvature, we have:

$$
\begin{aligned}
\widetilde{H}(x) & =\frac{1}{n+k} \sum_{i=1}^{n+k+1}\left\langle\widetilde{\nabla}_{\widetilde{E}_{i}} \widetilde{E}_{i}(x), \tilde{\eta}(x)\right\rangle=\frac{1}{n+k} \sum_{i=1}^{n+1}\left\langle\widetilde{\nabla}_{\widetilde{E}_{i}} \widetilde{E}_{i}(x), \tilde{\eta}(x)\right\rangle \\
& =\frac{1}{n+k}\left(\sum_{i=1}^{n+1}\left\langle l_{x}^{-1}\left(\nabla_{E_{i}} E_{i}(z)\right)+\frac{1}{2}\left(\left[\widetilde{E}_{i}, \widetilde{E}_{i}\right](x)\right)^{v}, \tilde{\eta}(x)\right\rangle\right) \\
& =\frac{1}{n+k} \sum_{i=1}^{n+1}\left\langle l_{x}^{-1}\left(\nabla_{E_{i}} E_{i}(z)\right), l_{x}^{-1}(\eta(x))\right\rangle \\
& =\frac{1}{n+k} \sum_{i=1}^{n}\left\langle\nabla_{E_{i}} E_{i}(z), \eta(z)\right\rangle=\frac{n}{n+k} H(z) .
\end{aligned}
$$

Proof of (ii). Choose $\tilde{v} \in T_{x} \tilde{M}$. We may then write

$$
\tilde{v}=l_{x}^{-1}(v)+w,
$$

where $v \in T_{z} M$ and $w$ is vertical. Then

$$
\begin{aligned}
\left\langle l_{x}^{-1}(\operatorname{grad} H(z)), \tilde{v}\right\rangle & =\left\langle l_{x}^{-1}(\operatorname{grad} H(z)), l_{x}^{-1}(v)+w\right\rangle=\left\langle l_{x}^{-1}(\operatorname{grad} H(z)), l_{x}^{-1}(v)\right\rangle \\
& =\langle\operatorname{grad} H(z), v\rangle=d(H)_{z}(v)=d g(H)_{z}\left(d \pi_{x}(\tilde{v})\right) \\
& =d(H \circ \pi)_{x}(\tilde{v})=d g\left(\frac{n+k}{n} \tilde{H}\right)_{x}(\tilde{v})=\frac{n+k}{n} d \widetilde{H}_{x}(\tilde{v}) .
\end{aligned}
$$

Therefore,

$$
\operatorname{grad} \widetilde{H}(x)=\frac{n}{n+k} l_{x}^{-1}(\operatorname{grad} H(z)) .
$$


Proof of (iii). As we have seen, $\left\langle\widetilde{\nabla}_{\widetilde{E}_{i}} \tilde{\eta}(x), \widetilde{E}_{j}(x)\right\rangle=0$ if $i, j \in\{n+2, \ldots, n+k+1\}$. Moreover,

$$
\begin{aligned}
\sum_{i, j=1}^{n+1}\left\langle\widetilde{\nabla}_{\widetilde{E}_{i}} \tilde{\eta}(x), \widetilde{E}_{j}(x)\right\rangle^{2} & =\sum_{i, j=1}^{n+1}\left\langle l_{x}^{-1}\left(\nabla_{E_{i}} \eta(z)\right)+\frac{1}{2}\left(\left[\widetilde{E}_{i}, \tilde{\eta}\right](x)\right)^{v}, \widetilde{E}_{j}(x)\right\rangle^{2} \\
& =\sum_{i, j=1}^{n+1}\left\langle l_{x}^{-1}\left(\nabla_{E_{i}} \eta(z)\right), l_{x}^{-1}\left(E_{j}(z)\right)\right\rangle^{2} \\
& =\sum_{i, j=1}^{n+1}\left\langle\nabla_{E_{i}} \eta, E_{j}\right\rangle^{2}=\|B\|^{2} .
\end{aligned}
$$

Assume that $i \in\{1, \ldots, n+1\}$ and $j \in\{n+2, \ldots, n+k+1\}$. We have, since $\widetilde{E}_{j}$ is a vector field on $\widetilde{M}$,

$$
\left\langle\widetilde{\nabla}_{\widetilde{E}_{i}} \tilde{\eta}(x), \widetilde{E}_{j}(x)\right\rangle=-\left\langle\tilde{\eta}(x), \widetilde{\nabla}_{\widetilde{E}_{i}} \widetilde{E}_{j}(x)\right\rangle .
$$

Set $F_{l}=d\left(L_{x}^{-1}\right)_{x}\left(\widetilde{E}_{l}\right), l=1, \ldots, n+k+1$. We then have

$$
\begin{aligned}
\left\langle\widetilde{\nabla}_{\widetilde{E}_{i}} \tilde{\eta}(x), \widetilde{E}_{j}(x)\right\rangle & =-\left\langle\tilde{\eta}(x), \widetilde{\nabla}_{d\left(L_{x}\right)_{e}\left(F_{i}\right)} d\left(L_{x}\right)_{e}\left(F_{j}\right)\right\rangle \\
& =-\frac{1}{2}\left\langle\tilde{\eta}(x),\left[d\left(L_{x}\right)_{e}\left(F_{i}\right), d\left(L_{x}\right)_{e}\left(F_{j}\right)\right](x)\right\rangle \\
& =-\frac{1}{2}\left\langle N(\pi(x)), d\left(R_{x}^{-1}\right)_{x}\left(\left[d\left(L_{x}\right)_{e}\left(F_{j}\right), d\left(L_{x}\right)_{e}\left(F_{j}\right)\right](x)\right)\right\rangle \\
& \left.=-\frac{1}{2}\left\langle N(\pi(x)),\left[\operatorname{Ad}_{x}\left(F_{i}\right), \operatorname{Ad}_{x}\left(F_{j}\right)\right](x)\right)\right\rangle \\
& =-\frac{1}{2}\left\langle\left[N\left(\pi(x), \operatorname{Ad}_{x}\left(F_{i}\right)\right], \operatorname{Ad}_{x}\left(F_{j}\right)\right\rangle\right. \\
& =-\frac{1}{2}\left\langle\operatorname{ad}_{N(\pi(x)}\left(\operatorname{Ad}_{x}\left(F_{i}\right)\right), \operatorname{Ad}_{x}\left(F_{j}\right)\right\rangle .
\end{aligned}
$$

Note that

$$
\operatorname{Ad}_{x}\left(F_{l}\right)=d\left(R_{x}^{-1}\right)_{x} d\left(L_{x}\right)_{e}\left(d\left(L_{x}^{-1}\right)_{x}\left(\widetilde{E}_{l}\right)=d\left(R_{x}^{-1}\right)_{x}\left(\widetilde{E}_{l}\right),\right.
$$

so that, if $l=i$,

$$
\operatorname{Ad}_{x}\left(F_{l}\right)=\operatorname{Ad}_{x}\left(F_{i}\right)=\Gamma_{z}\left(E_{i}\right)
$$

We then have

$$
\left\langle\widetilde{\nabla}_{\widetilde{E}_{i}} \tilde{\eta}(x), \widetilde{E}_{j}(x)\right\rangle=-\frac{1}{2}\left\langle\operatorname{ad}_{N(\pi(x)}\left(\Gamma_{z}\left(E_{i}\right)\right), \operatorname{Ad}_{x}\left(F_{j}\right)\right\rangle .
$$

Moreover, $\left\{\operatorname{Ad}_{x}\left(F_{j}\right)\right\}=\left\{d\left(R_{x}^{-1}\right)_{x}(\widetilde{E j})\right\}_{j=n+2, \ldots, n+k+1}$ is an orthonormal basis of H. In particular,

$$
\left\langle\widetilde{\nabla}_{\widetilde{E}_{i}} \tilde{\eta}(x), \widetilde{E}_{j}(x)\right\rangle=-\frac{\sqrt{2}}{2}\left\langle B_{z}^{i}\left(E_{i}\right), d\left(R_{x}^{-1}\right)_{x}(\widetilde{E j})\right\rangle,
$$


and thus

$$
\sum_{\substack{i=1, \ldots, n+1 \\ j=n+2, \ldots, n+k+1}}\left\langle\widetilde{\nabla}_{\widetilde{E}_{i}} \tilde{\eta}(x),\left.\widetilde{E}_{j}(x)\right|^{2}=\frac{1}{2}\left\|B_{z}^{i}\right\|^{2} .\right.
$$

Then

$$
\begin{aligned}
\|\widetilde{B}(x)\|^{2} & =\sum_{i, j=1}^{n+k+1}\left\langle\widetilde{\nabla}_{\widetilde{E}_{i}} \tilde{\eta}(x), \widetilde{E}_{j}(x)\right\rangle^{2} \\
& =\sum_{i, j=1}^{n+1}\left\langle\widetilde{\nabla}_{\widetilde{E}_{i}} \tilde{\eta}(x), \widetilde{E}_{j}(x)\right\rangle^{2}+\sum_{i, j=n+2}^{n+k+1}\left\langle\widetilde{\nabla}_{\widetilde{E}_{i}} \tilde{\eta}(x), \widetilde{E}_{j}(x)\right\rangle^{2}+2 \sum_{\substack{i=1, \ldots, n+1 \\
j=n+2, \ldots, n+k+1}}\left\langle\widetilde{\nabla}_{\widetilde{E}_{i}} \tilde{\eta}(x), \widetilde{E}_{j}(x)\right\rangle^{2} \\
& =\|B\|^{2}+\left\|B^{i}\right\|^{2} .
\end{aligned}
$$

Keeping the same meanings for $B, B^{i}, H$ and $\operatorname{grad} H$ as above, we have:

Theorem 4.3. Let $M$ be an immersed orientable hypersurface of $\mathbb{G} / \mathbb{M}$, and let $N: M \rightarrow \mathbb{S}^{n+k}$ be the Gauss map of $M$. Then

$$
\text { (4-2) } \Delta N(z)=-n \Gamma_{z}(\operatorname{grad} H(z))-\left(\|B\|^{2}+\left\|B^{i}\right\|^{2}+(n+k) \operatorname{Ric}\left(l_{x}^{-1}(\eta(z))\right)\right) N(z)
$$

for all $z \in M$ and $x \in \pi^{-1}(z)$, where $\eta$ is a unit normal vector field to $M$ and $\operatorname{Ric}(w)$ the Ricci curvature of $\mathbb{G}$ with respect to $w \in T \mathbb{G}$.

Proof. As before, set $\tilde{\eta}=l_{x}^{-1}(\eta)$. As mentioned, $\tilde{\eta}$ is a unitary normal vector field to $\widetilde{M}=\pi^{-1}(M)$. Let $\widetilde{N}: \widetilde{M} \rightarrow \mathbb{S}^{n+k}$ be the Gauss map of $\widetilde{M}$ :

$$
\tilde{N}(x)=d\left(R_{x}^{-1}\right)_{x}(\tilde{\eta}(x)) .
$$

We claim that, if $z=\pi(x)$,

$$
\Delta_{M} N(z)=\Delta_{\widetilde{M}} \tilde{N}(x) .
$$

To see this, note that $N(z)=\Gamma_{z}(\eta(z))=d\left(R_{x}\right)_{x}^{-1}(\tilde{\eta}(x))=\tilde{N}(x)$, so writing

$$
N(z)=\sum_{i=1}^{n+k+1} N_{i}(z) e_{i} \quad \text { and } \quad \tilde{N}(x)=\sum_{i=1}^{n+k+1} \tilde{N}_{i}(x) e_{i}
$$

in some orthonormal basis $\left\{e_{i}\right\}_{i=1 \ldots, n+k+1}$ of $\mathscr{G}$, we have

$$
\tilde{N}_{i}(x)=N_{i}(z)=\left(N_{i} \circ \pi\right)(x),
$$

where $x \in \pi^{-1}(z)$. By Lemma 4.1, we have

$$
\Delta_{\widetilde{M}} \widetilde{N}_{i}(x)=\left(\Delta_{M} N_{i}\right) \circ \pi(x)=\Delta_{M} N_{i}(z),
$$


proving (4-3). It follows by Theorem 1 of [Espírito-Santo et al. 2003] that

$$
\Delta_{\widetilde{M}} \tilde{N}(x)=-(n+k) d\left(R_{x}^{-1}\right)_{x}(\operatorname{grad} \widetilde{H}(x))-\left(\|\widetilde{B}\|^{2}+(n+k) \operatorname{Ric} \tilde{\eta}(x)\right) \tilde{N}(x)
$$

so that, applying Lemma 4.2, we obtain

$$
\begin{aligned}
\Delta N(z)= & -(n+k) d\left(R_{x}^{-1}\right)_{x}\left(\frac{n}{n+k} l_{x}^{-1}(\operatorname{grad} H(z))\right) \\
& -\left(\|\widetilde{B}\|^{2}+(n+k) \operatorname{Ric}(\tilde{\eta}(x))\right) N(z) \\
= & -n \Gamma_{z}(\operatorname{grad} H(z))-\left(\|B\|^{2}+\left\|B^{i}\right\|^{2}+(n+k) \operatorname{Ric}\left(l_{x}^{-1}(\eta(z))\right)\right) N(z),
\end{aligned}
$$

proving the theorem.

Theorem 4.3 has an application to constant mean curvature immersions:

Corollary 4.4. Let $M$ be an orientable hypersurface in $\mathbb{G} / \mathbb{H}$ and $N: M \rightarrow \mathbb{S}^{n+k}$ the Gauss map of $M$. There is equivalence between:

(i) $M$ has constant mean curvature.

(ii) The Gauss map $N: M^{n} \rightarrow \mathbb{S}^{n+k}$ is harmonic.

(iii) $N$ satisfies the equation

$$
\Delta N(z)=-\left(\|B\|^{2}+\left\|B^{i}\right\|^{2}+(n+k) \operatorname{Ric}\left(l_{x}^{-1}(\eta(z))\right)\right) N(z) .
$$

We apply the definition of a $\left(1 / 2^{l}\right)$-sphere (page 46$)$ to a result that extends Corollary 2 of [Espírito-Santo et al. 2003]:

Theorem 4.5. Let $M$ be a compact constant mean curvature hypersurface of $\mathbb{G} / \mathbb{W}$ and $N: M \rightarrow \mathbb{S}^{n+k}$ the Gauss map of $M$. Assume that $M$ is not totally geodesic, or that $\left\|B^{i}\right\|>0$, or that Ric $>0$. Given $l \in \mathbb{N}$, let

$$
\mathbb{S}^{n+k, l}=\bigcap_{i=1}^{l}\left(\mathbb{S}_{i}^{n+k}\right)_{+}
$$

be a $\left(1 / 2^{l}\right)$-sphere of $\mathbb{S}^{n+k}$. There is equivalence between:

(i) $N(M) \subset \mathbb{S}^{n+k, l}$.

(ii) $N(M) \subset \bigcap_{i=1}^{l} \partial\left(\mathbb{S}_{i}^{n+k}\right)_{+}$.

(iii) $\mathscr{K}:=\left(\bigcap_{i=1}^{l} \partial\left(\mathbb{S}_{i}^{n+k}\right)_{+}\right)^{\perp}$ is a Lie subalgebra of $\mathscr{G}$ and $M$ is invariant under the Lie subgroup $\mathbb{K}$ of $\mathbb{G}$ whose Lie algebra is $\mathscr{K}$.

Proof. We first prove that (i) implies (ii). Given $i$, we have $N(M) \subset H_{i}$, where $H_{i}$ is the half-space of $\mathscr{G}$ such that $\mathbb{S}_{i}^{n+k}=H_{i} \cap \mathbb{S}^{n+k}$. Therefore, there is $v \in \mathbb{S}^{n+k}$ orthogonal to $H_{i}$ such that $\langle N(z), v\rangle \leq 0$ for all $z \in M$. Using (4-4), and considering that Ric $\geq 0$, we obtain

$$
\Delta\langle N, v\rangle=\langle\Delta N, v\rangle=-\left(\|B\|^{2}+\left\|B^{i}\right\|+(n+k) \operatorname{Ric}\left(l_{x}^{-1}(\eta(z))\right)\right)\langle N(z), v\rangle \geq 0
$$


so that $\langle N, v\rangle$ is a subharmonic function on the compact manifold $M$. It follows that $\langle N, v\rangle$ is constant and then $\Delta\langle N, v\rangle=0$. Hence, since either $\|B\|>0,\left\|B^{i}\right\|>0$, or $\operatorname{Ric}\left(l_{x}^{-1}(\eta(z))\right)>0$, we have $\langle N, v\rangle=0$, so that $N(M) \subset \partial H_{i}$. This proves that (i) implies (ii). The implication (iii) $\Rightarrow$ (ii) follows from Proposition 3.4, and (ii) $\Rightarrow$ (i) is obvious.

Remark. If $M$ is admitted to be totally geodesic, $\left\|B^{i}\right\|=0$, and $\operatorname{Ric}\left(l_{x}^{-1}(\eta(z))\right)=0$, the theorem is false. Planes in $\mathbb{R}^{3}$ give counterexamples to the theorem.

Corollary 4.6. Assume that $M$ is not totally geodesic, or $\left|B^{i}\right|>0$, or Ric $>0$. Let $M$ be a compact constant mean curvature hypersurface of $\mathbb{G} / \mathbb{H}$ and $N: M \rightarrow \mathbb{S}^{n+k}$ the Gauss map of $M$. Let $\mathbb{S}_{l}^{n+k}$ be a $\left(1 / 2^{l}\right)$-sphere of $\mathbb{S}^{n+k}$. Assume that $l \geq n$ and

$$
N(M) \subset \mathbb{S}_{l}^{n+k} .
$$

Then $M$ is an extrinsically homogeneous submanifold of $\mathbb{G} / \mathbb{W}$ (recall that $\mathbb{G} / \mathbb{W}$ has dimension $n+1)$.

To investigate if a constant mean hypersurface $M$ is invariant under a specific Lie subgroup of isometries, we shall make use of the following result:

Corollary 4.7. Let $M$ be a compact constant mean curvature hypersurface of $\mathbb{G} / \mathbb{H}$ and $N: M \rightarrow \mathbb{S}^{n+k}$ the Gauss map of $M$. Assume that $M$ is not totally geodesic, or $\left|B^{i}\right|>0$, or Ric $>0$. Let $\mathbb{K}$ be a Lie subgroup of $\mathbb{G}$ of dimension $l$ and $\mathscr{Y}$ the Lie algebra of $\mathbb{K}$. Let $\mathcal{M}$ be the orthogonal complement of $\mathscr{Y}$ in $\mathscr{G}$. Let $\left(\mathbb{S}_{1}^{n+k}\right)_{+}, \ldots,\left(\mathbb{S}_{l}^{n+k}\right)_{+}$be l half-spheres of $\mathbb{S}^{n+k}$ such that $\mathcal{M} \cap \mathbb{S}^{n+k} \subset\left(\mathbb{S}_{i}^{n+k}\right)_{+}$ for $i=1, \ldots, l$, that is,

$$
\mu \cap \mathbb{S}^{n+k}=\bigcap_{i=1}^{l} \partial\left(\mathbb{S}_{i}^{n+k}\right)_{+} .
$$

Then $M$ is $\mathbb{K}$-invariant if and only if

$$
N(M) \subset \bigcap_{i=1}^{l}\left(\mathbb{S}_{i}^{n+k}\right)_{+} .
$$

Corollary 4.8. Assume that $\mathbb{G} / \mathbb{M}$ is a two-point homogeneous manifold (that is, one such that $\mathbb{G}=\operatorname{Iso}(\mathbb{G} / \mathbb{G}))$. Let $M$ be a compact constant mean curvature hypersurface of $\mathbb{G} / \mathbb{W}$ and $N: M \rightarrow \mathbb{S}^{n+k}$ the Gauss map of $M$. Let $M$ be the orthogonal complement of the Lie algebra $\mathscr{H}$ of $\mathbb{H}$ in $\mathcal{G}_{\text {. Let }}\left(\mathbb{S}_{1}^{n+k}\right)_{+}, \ldots,\left(\mathbb{S}_{l}^{n+k}\right)_{+}$ be $l$ half-spheres of $\mathbb{S}^{n+k}$ such that $\mathcal{M} \cap \mathbb{S}^{n+k} \subset\left(\mathbb{S}_{i}^{n+k}\right)_{+}$for $i=1, \ldots$, l. Then

$$
N(M) \subset \bigcap_{i=1}^{l}\left(\mathbb{S}_{i}^{n+k}\right)_{+}
$$

if and only if $M$ is a geodesic sphere. 
Proof. In a two-point homogeneous space the isotropy subgroup $\mathbb{U}$ of $\mathbb{G}$ acts transitively on geodesic spheres; now apply Corollary 4.7.

Theorem 4.9. Assume that $\operatorname{dim} \mathbb{G} / \mathbb{H}=3$. Let $M$ be a complete constant mean curvature surface of $\mathbb{G} / \mathbb{H}$ and $N: M \rightarrow \mathbb{S}^{3+k}$ the Gauss map of $M$. Assume that Ric $>0$ or $\left\|B^{i}\right\|>0$ or $M$ is not totally geodesic. Then $N(M)$ is contained in a hemisphere of the unit sphere in $G$ if and only if $M$ is invariant under a oneparameter subgroup of isometries of $\mathbb{G}$.

Proof. If $M$ is invariant under a one-parameter subgroup of isometries of $\mathbb{G} / \mathbb{W}$ determined by a $X \in \mathscr{G}$ then $N(M)$ is contained in a hemisphere whose boundary hyperplane is orthogonal to $X$. The other direction follows from Corollary 2 of [Fornari and Ripoll 2004], since given $w \in N(M)^{\perp}, \zeta(w)$ is a Killing field of $\mathbb{G} / \mathbb{W}$ such that

$$
f(z):=\langle\zeta(w)(z), \eta(z)\rangle
$$

does not change sign on $M$; however, for completeness, we give the details of the proof, which is essentially the same as that of Theorem 1 of [Hoffman et al. 1982].

Let $(\widehat{M}, \pi)$ be the universal covering of $M$. First assume that $\widehat{M}$ is the disk and suppose that $f \leq 0$. Then, noting that $f(z)=\langle w, N(z)\rangle$, we obtain from (4-4)

$$
\Delta f=\langle w, \Delta N\rangle=-\left(\|B\|^{2}+\left\|B^{i}\right\|^{2}+(n+k) \operatorname{Ric}\left(l_{x}^{-1}(\eta(z))\right)\right) f \geq 0,
$$

so that $f$ is subharmonic. Therefore, if $f$ vanishes at some point of $M$, it vanishes identically (by the maximum principle), so $M$ is invariant under $\zeta(w)$, proving the theorem in this case. We now show that the case $f<0$ leads to a contradiction. By the Gauss equation, we have

$$
\|B\|^{2}=4 H^{2}-2(K-\widetilde{K}),
$$

where $K$ is the Gaussian curvature of $M$ and $\widetilde{K}$ the sectional curvature of $\mathbb{G} / \mathbb{H}$ on tangent planes of $M$. From the second equality in (4-5) and from (4-6) we obtain

$$
\Delta f-2 K f+\left(\left\|B^{i}\right\|^{2}+(n+k) \operatorname{Ric}\left(l_{x}^{-1}(\eta(z))\right)+2 \widetilde{K}+4 H^{2}\right) f=0 .
$$

However, (4-7) contradicts [Fischer-Colbrie and Schoen 1980, Corollary 3], which states that when $K$ is the Gaussian curvature of a complete conformal metric on the unit disk there can be no negative solution of equation (4-7) if

$$
\left\|B^{i}\right\|^{2}+(n+k) \operatorname{Ric}\left(l_{x}^{-1}(\eta(z))\right)+2 \widetilde{K}+4 H^{2} \geq 0
$$

(note that $\widetilde{K} \geq 0$ and Ric $\geq 0$ ).

It follows that $\widehat{M}$ is the sphere or the plane, proving the assertion stated above. Thus $f \circ \pi$ is a subharmonic and bounded, hence constant, function on $\mathbb{R}^{2}$; thus $f$ is constant. Then $\Delta f=0$ and $\left(\|B\|^{2}+\left\|B^{i}\right\|^{2}+(n+k) \operatorname{Ric}\left(l_{x}^{-1}(\eta(z))\right)\right) f=0$, and the conclusion of the theorem is now immediate. 
We have the following stability criterion, whose proof is the same as that of [Espírito-Santo et al. 2003, Theorem 5]:

Theorem 4.10. Assume that $\operatorname{dim}(\mathbb{G} / \mathbb{M})=3$ and let $M$ be a constant mean curvature surface in $\mathbb{G} / \mathbb{H}$. Let $D \subset M$ be a domain such that $\bar{D}$ lies in the interior of $M$. If $N(\bar{D})$, the spherical image of $\bar{D}$, is contained in a open hemisphere of the unit sphere in $G$, then $\bar{D}$ is stable.

In the most common cases $\mathbb{G}$ has constant Ricci curvature. This always occurs when the Killing form of $\mathbb{G}$ is negative definite, as it is shown below. The Killing form of $\mathbb{G}$ is negative definite, for instance, when $\mathbb{G}$ is compact and has finite center.

Lemma 4.11. Suppose that the Killing form of $\mathbb{G}$ is negative definite. Then any bi-invariant metric $\langle$,$\rangle satisfies$

$$
\operatorname{Ric}(u, v)=\frac{1}{4}\langle u, v\rangle,
$$

where $u$ and $v$ are tangent vectors to $\mathbb{G}$. In particular, $\mathbb{G}$ has constant Ricci curvature $\frac{1}{4}$.

Proof. the Killing form $B$ of $\mathbb{G}$ on the Lie algebra $\mathscr{G}$ is given by

$$
B(u, v)=\operatorname{trace}\left(\operatorname{ad}_{u} \circ \operatorname{ad}_{v}\right) \quad \text { for } u, v \in \mathscr{G},
$$

and we have assumed it to be negative definite. Consider any bi-invariant metric $\langle$,$\rangle on \mathbb{G}$ and let $\left\{E_{j}\right\}_{j=1, \ldots, n+k+1}$ be an orthonormal basis of $\mathscr{G}$ with respect to $\langle$,$\rangle . Given x \in \mathbb{G}$ and $u, v \in \mathscr{G}$, we have

$$
\begin{aligned}
\operatorname{Ric}(u, v) & =\sum_{j=1}^{n+k+1}\left\langle R\left(u, E_{j}\right) v, E_{j}\right\rangle=\frac{1}{4} \sum_{j=1}^{n+k+1}\left\langle\left[\left[u, E_{j}\right], v\right], E_{j}\right\rangle \\
& =-\frac{1}{4} \sum_{j=1}^{n+k+1}\left\langle\left[E_{j}, v\right],\left[u, E_{j}\right]\right\rangle=\frac{1}{4} \sum_{j=1}^{n+k+1}\left\langle\left[E_{j}, v\right],\left[E_{j}, u\right]\right\rangle \\
& =\frac{1}{4} \sum_{j=1}^{n+k+1}\left\langle\operatorname{ad}_{E_{j}}(v), \operatorname{ad}_{E_{j}}(u)\right\rangle=-\frac{1}{4} \sum_{j=1}^{n+k+1}\left\langle\left(\operatorname{ad}_{E_{j}}\right)^{2}(v), u\right\rangle \\
& \left.=-\frac{1}{4} \sum_{j=1}^{n+k+1}\left(\operatorname{ad}_{E_{j}}\right)^{2}(v), u\right\rangle,
\end{aligned}
$$

where in the third and sixth equalities we used the skew-symmetry of $\operatorname{ad}_{X}$ :

$$
\left\langle\operatorname{ad}_{X}(u), v\right\rangle=-\left\langle\operatorname{ad}_{X}(v), u\right\rangle .
$$


Considering the linear symmetric transformation

$$
T:=\sum_{j=1}^{n+k+1}\left(\operatorname{ad}_{E_{j}}\right)^{2},
$$

we obtain, for all $u, v \in \mathscr{G}$,

$$
\begin{aligned}
\langle T(u), v\rangle & =\left\langle\sum_{j=1}^{n+k+1}\left(\operatorname{ad}_{E_{j}}\right)^{2}(u), v\right\rangle=-\sum_{j=1}^{n+k+1}\left\langle\operatorname{ad}_{E_{j}}(u), \operatorname{ad}_{E_{j}}(v)\right\rangle \\
& =-\sum_{j=1}^{n+k+1}\left\langle\operatorname{ad}_{u}\left(E_{j}\right), \operatorname{ad}_{v}\left(E_{j}\right)\right\rangle=\sum_{j=1}^{n+k+1}\left\langle E_{j}, \operatorname{ad}_{u} \circ \operatorname{ad}_{v}\left(E_{j}\right)\right\rangle \\
& =\operatorname{trace}\left(\operatorname{ad}_{u} \circ \operatorname{ad}_{v}\right)=B(u, v) .
\end{aligned}
$$

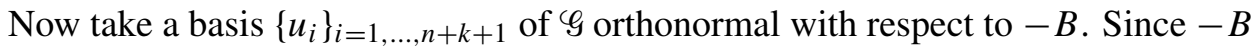
is positive definite, we obtain $\left\langle T\left(u_{i}\right), u_{j}\right\rangle=-\delta_{i j}$, so $T=-I$, where $I$ denotes the

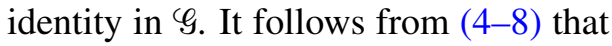

$$
\operatorname{Ric}(u, v)=\frac{1}{4}\langle u, v\rangle,
$$

concluding the proof of the lemma.

We obtain from Theorem 4.3 and this last lemma:

Corollary 4.12. Under the hypothesis of Theorem 4.3, assume moreover that the Killing form of $\mathbb{G}$ is negative definite. Then

$$
\Delta N(z)=-n \Gamma_{z}(\operatorname{grad} H(z))-\left(\|B\|^{2}+\left\|B^{i}\right\|^{2}+\frac{1}{4}(n+k)\right) N(z) .
$$

We now apply the previous results to the special case of $\mathbb{S}^{3}=\mathrm{SO}(4) / \mathrm{SO}(3)$. Recall that the Lie algebra $\mathfrak{s o}(4)$ of $\mathrm{SO}(4)$ is given by the $4 \times 4$ matrices $Z$ satisfying $Z^{t}=-Z$. We may consider in $\mathfrak{s o ( 4 )}$ the Killing form

$$
\langle X, Y\rangle=-\frac{1}{4} \operatorname{trace}\left(X Y^{t}\right), X, Y \in \mathfrak{s o}(4),
$$

although the result, of course, is independent of the bi-invariant Riemannian metric considered.

Given $\alpha \in \mathbb{R}$, let $\left\{\phi_{t}^{\alpha}\right\}_{t \in \mathbb{R}}$ be the one-parameter subgroup of isometries of $\mathbb{S}^{3}$ defined by

$$
\phi_{t}^{\alpha}=\left[\begin{array}{cccc}
\cos t & \sin t & 0 & 0 \\
-\sin t & \cos t & 0 & 0 \\
0 & 0 & \cos \alpha t & \sin \alpha t \\
0 & 0 & -\sin \alpha t & \cos \alpha t
\end{array}\right]
$$


Corollary 4.13. Let $M$ be an immersed orientable complete surface with constant mean curvature in $\mathbb{S}^{3}=\mathrm{SO}(4) / \mathrm{SO}(3)$ with Gauss map $N: M \rightarrow \mathbb{S}^{5}$. Then $N(M)$ is contained in $\mathbb{S}_{+}^{5}$ if and only if, up to an isometry, $M$ is $\phi_{t}^{a}$-invariant for some $\alpha \in \mathbb{R}$.

Proof. One direction is obvious. For the other, note that Theorem 4.9 implies that $M$ is invariant under a one-parameter Lie subgroup $\phi_{t}$ of isometries of $\mathbb{S}^{3}$ of the form $\exp (t X)$ for some nonzero vector $X \in \mathfrak{s o}(4)$. By the maximal torus theorem, there are $\delta, \varepsilon \in \mathbb{R}$ and $g \in \mathrm{SO}(4)$ such that

$$
\operatorname{Ad}_{g} X=\left[\begin{array}{rrrr}
0 & \delta & 0 & 0 \\
-\delta & 0 & 0 & 0 \\
0 & 0 & 0 & \varepsilon \\
0 & 0 & -\varepsilon & 0
\end{array}\right] .
$$

We may assume without loss of generality that $\delta \neq 0$ so that the one-dimensional Lie subalgebra generated by $\operatorname{Ad}_{g} X$ is the same as the one generated by

$$
X_{\alpha}=\left[\begin{array}{rrrr}
0 & 1 & 0 & 0 \\
-1 & 0 & 0 & 0 \\
0 & 0 & 0 & \alpha \\
0 & 0 & -\alpha & 0
\end{array}\right],
$$

whose associated Lie subgroup is $\phi_{t}^{\alpha}$. It follows that $M$ is congruent to $g(M)$ and $g(M)$ is $\phi_{t}^{\alpha}$-invariant.

An open question on the study of minimal surfaces in the sphere $\mathbb{S}^{3}$ is whether there exist embedded minimal tori in $\mathbb{S}^{3}$ besides the Clifford tori. Lawson conjectured that the answer is no. We may apply the corollary above to obtain the following characterization of the Clifford torus and of the surfaces of revolution of $\mathbb{S}^{3}$ as well.

Observe that $\phi_{t}^{0}$ is a rotational one-parameter subgroup of isometries of $\mathbb{S}^{3}$ and that $\phi_{t}^{1}$ is the Hopf action. Given $\alpha \in \mathbb{R}$, denote by $\mathbb{S}_{\alpha}^{5}$ the half-sphere of $\mathbb{S}^{5}$ in $\mathfrak{s o}(4)$ whose boundary is orthogonal to $X_{\alpha}$.

Corollary 4.14. Let $M$ be an immersed orientable complete surface with constant mean curvature in $\mathbb{S}^{3}=\mathrm{SO}(4) / \mathrm{SO}(3)$ and let $N: M \rightarrow \mathbb{S}^{5}$ be the Gauss map of $M$.

(1) $N(M) \subset \mathbb{S}_{1}^{5}$ if and only if $M$ is a Clifford torus.

(2) $N(M) \subset \mathbb{S}_{0}^{5}$ if and only if $M$ is a surface of revolution.

For the proof of (a) we use the fact that a complete cmc surface in $\mathbb{S}^{3}$ which is invariant under the Hopf action is a Clifford torus. This is easy to prove using an argument from the so-called equivariant geometry (see the proof of Theorem 4 of [Espírito-Santo et al. 2003]). 
Remark. Surfaces of revolution with $\mathrm{cmc}$ in $\mathbb{S}^{3}$ have been described in many places. A description of these surfaces using a special coordinate system is given in [Fornari and Ripoll 2004].

\section{References}

[Eells and Sampson 1964] J. Eells, Jr. and J. H. Sampson, "Harmonic mappings of Riemannian manifolds", Amer. J. Math. 86 (1964), 109-160. MR 29 \#1603 Zbl 0122.40102

[Espírito-Santo et al. 2003] N. do Espírito-Santo, S. Fornari, K. Frensel, and J. Ripoll, "Constant mean curvature hypersurfaces in a Lie group with a bi-invariant metric", Manuscripta Math. 111:4 (2003), 459-470. MR 2004f:53072

[Fischer-Colbrie and Schoen 1980] D. Fischer-Colbrie and R. Schoen, "The structure of complete stable minimal surfaces in 3-manifolds of nonnegative scalar curvature", Comm. Pure Appl. Math. 33:2 (1980), 199-211. MR 81i:53044 Zbl 0439.53060

[Fornari and Ripoll 2004] S. Fornari and J. Ripoll, "Killing fields, mean curvature, translation maps", Illinois J. Math. 48:4 (2004), 1385-1403. MR 2005i:53037 Zbl 02140243

[Hoffman et al. 1982] D. A. Hoffman, R. Osserman, and R. Schoen, "On the Gauss map of complete surfaces of constant mean curvature in $\mathbb{R}^{3}$ and $\mathbb{R}^{4}$,, Comment. Math. Helv. 57:4 (1982), 519-531. MR 84f:53004 Zbl 0512.53008

Received June 1, 2004.

FIDELIS BITTENCOURT

Universidade Federal de Santa Maria

CCNE - DEPARTAMENTO DE MATEMÁticA

CAMPUS CAMOBI

97105-900 SANTA MARIA, RS

BRAZIL

fidelis@smail.ufsm.br

JAIME RIPOLL

UNIVERSIDADE FEDERAL DO RIO GRANDE DO SUL

InSTITUTO DE MATEMÁtica

Av. BENTO GONÇALVES 9500

91501-970 PORTO AlEGRE, RS

BRAZIL

ripoll@mat.ufrgs.br 